\title{
Dynamic Analysis and CFD Numerical Simulation on Backpressure Filling System
}

\author{
Jing Qian, ${ }^{1,2}$ J. P. Lu, ${ }^{1,2}$ S. L. Hui, ${ }^{1}$ Y. J. Ma, ${ }^{1}$ and D. Y. Li ${ }^{1}$ \\ ${ }^{1}$ School of Mechanical Engineering, Jiangnan University, Wuxi, Jiangsu 214122, China \\ ${ }^{2}$ Jiangsu Key Laboratory of Advanced Food Manufacturing Equipment and Technology, Jiangsu 214122, China \\ Correspondence should be addressed to Jing Qian; qj639@163.com
}

Received 27 June 2014; Revised 5 December 2014; Accepted 15 December 2014

Academic Editor: Kit Keith L. Yam

Copyright (c) 2015 Jing Qian et al. This is an open access article distributed under the Creative Commons Attribution License, which permits unrestricted use, distribution, and reproduction in any medium, provided the original work is properly cited.

\begin{abstract}
A backpressure filling system is a kind of air type filling system which could be applied to power type, fine or coarse grain, or mixtures with fine and coarse components. The working principle of backpressure filling system was discussed based on fundamental hydromechanics. The research limit values of backpressure were achieved via mechanical analysis. Comparing with the exit velocity of material by theoretical analysis and numerical simulation, the CFD simulation model was confirmed and its related parameters were determined. The CFD numerical simulation shows the relationship between production capacity of packaging machine and backpressure, and the results matched actual operation of the equipment well. Combining with the demand of device capacity, the range of backpressure could be controlled at $8 \mathrm{kPa} 11 \mathrm{kPa}$.
\end{abstract}

\section{Introduction}

The key packaging technology of bulk material is the design of filling system. The typical filling system in industry includes screw type and impeller type. These two kinds of filling systems push the bulk material to filling spouts with high speed via screw blade and impeller blade, respectively. Their construction is simple and obtained material flow is stabilized. The weakness of two-type filling systems is that motors have to start and pause frequently, and power consumption is high. Moreover, abrasion of blade is faster, and the filling system is easy to be blocked, especially filling bulk material with particle, such as dry mortar.

Mortar is a kind of cementing building material and is composed of sand and cementing material (such as cement, lime putty, and clay). More customers want to buy matched dry mortar for their convenience. Adapting to the requirement of packaging filling for particle mixture material, reducing the abrasion of filling system and the incidence rate of industrial accident, the research group developed the backpressure filling system as shown in Figure 1 [1].

Backpressure filling system also can be named as fluidization filling system. It sets aeration pads on the wall of filling box near the filling spout. It provides fixed pressure on the bulk material which is in the filling bin, to prevent arched material and accelerate its flow. The advantages of backpressure filling system are that there are no mechanical vulnerable parts and there is less mechanical failure. Even so, if the pressure of backpressure cannot be controlled very well, a series of problems will take place. When the pressure is too high, air will break through the bulk material and go into the packing bag to bulge the bag. When the pressure is too low, the capacity of packaging machine will be insufficient.

The speed of bulk material near the exit of packaging machine could directly affect the capacity of the machine. Therefore, improving the flow velocity of exit could effectively increase the machine capacity. The impact coefficients on flow velocity include material properties, geometrical shape of filling box, and pressure of backpressure. The pressure of backpressure is one of the principal coefficients.

The value range of backpressure will affect regular work of the packaging machine, and it is crucial coefficient for the capacity of the equipment. The relationship between backpressure and equipment capacity is the fundamental mechanism research for industry application. The research work started at the limiting value of the backpressure. 


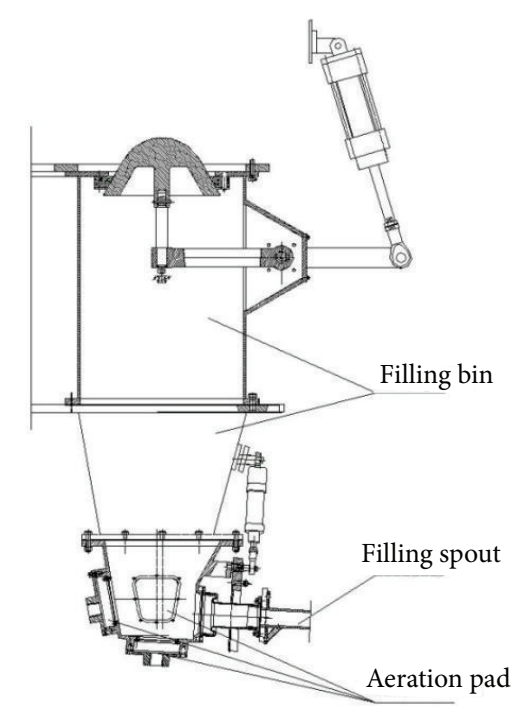

FIGURE 1: The backpressure filling system.

\section{Limit Pressure Value of Backpressure Filling System}

The force of bulk material to filling bin wall and the force of filling bin to bulk material are a pair of action and reaction. The pressure value which aeration pads provided should be between the dynamic pressure $P_{1}$ of bulk material to bin wall when the flow of bulk material is in steady state and breakthrough pressure $P_{2}$. If the backpressure is less than $P_{1}$, the thrust which aeration pads provided is limited, cannot accelerate bulk material's flow, and causes the slowdown of flow velocity of bulk material at exit. In this case, the capacity of packaging machine is limited. If the backpressure is greater than $P_{2}$, air will break through bulk material, and so the flow of bulk material becomes turbulent and ejection velocity of bulk material is unstable to affect the packing accuracy. Furthermore, amount of air will get into packing bag, thus causing the expansion or breakage of bag. Only when the backpressure is between $P_{1}$ and $P_{2}$, aeration pads could accelerate the flow of bulk material, and no adverse effects will arise.

2.1. Dynamic Pressure of Bulk Material on Bin Wall. The real filling bin was simplified to a cylinder bin as shown in Figure 2. The open mouth in the bottom is the exit, and bulk material flows out via the exit under the effect of gravity.

Assume that the flow of bulk material is in a constant state; that is, the bulk material is flowing into filling bin unceasingly and is flowing out from the exit. The outflow volume is equal to inflow volume. In this case, the dynamic pressure of bulk material to bulkhead has nothing to do with the time [2].

To find the equilibrium condition on dynamic pressure of bulk material to bin wall, researcher picks the infinitesimal element of filling bin to study as shown in Figure 2. Forces on this infinitesimal element are self-gravity, vertical pressures on top and bottom surface, horizontal pressure, and force of

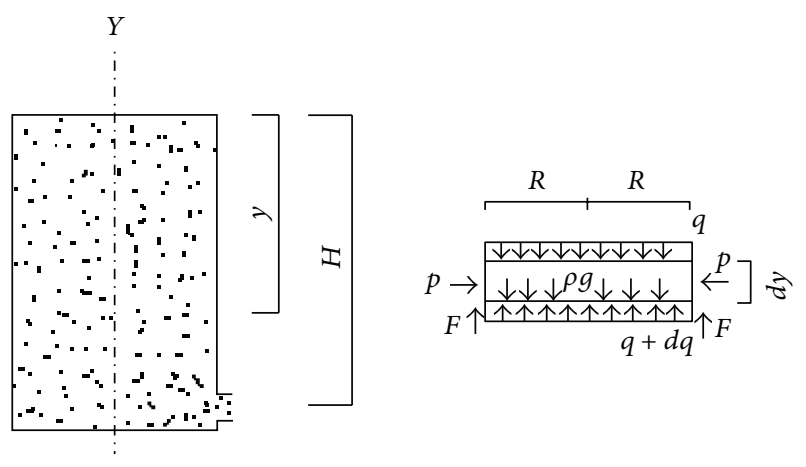

FIGURE 2: Filling bin and its force analysis.

friction $[3,4]$. According to the equilibrium of forces, the following equation could be achieved:

$$
\rho g-\frac{d q}{d y}-\frac{2 f p}{R}=0,
$$

where $p=k(y) q$.

In the above formula, $q$ is the vertical stress, $p$ is the horizontal stress, $f$ is the friction coefficient between the bulk material and the bulkhead, $\rho$ is the density of the bulk material, $R$ is the radius of the filling bin, $g$ is the gravitational acceleration, and $k(y)$ is lateral pressure coefficient, respectively. When the flow of material is in the steady state, the lateral pressure coefficient $k(y)$ varies by depth. Therefore the following equation could be achieved:

$$
\frac{d q}{d y}+\frac{2 k(y) f}{R} q=\rho g .
$$

Equation (2) is the dynamic pressure differential equation of bulkhead when the material flows inside, which is also a nonhomogeneous first-order linear differential equation. The general solution of equation is as follows:

$$
q=e^{-(2 f / R) \int k(y) d y}\left[\rho g \int e^{(2 f / R) \int k(y) d y} d y+c\right] .
$$

Due to the different deformation state when the bulk material flows in the filling bin, the stress state is different. When the bulk material is in the top of filling bin, the material is in the outward deformation state away from the vertical axis, which is also called the active stress state. The lateral pressure coefficient should be given as follows:

$$
K_{a}=\frac{1-\sin \phi_{i}}{1+\sin \phi_{i}}
$$

where $\phi_{i}$ is the internal friction angle of bulk material.

In the end of filling bin, the material is in the inward deformation state which is forward to vertical axis, and thus the material here is accordingly in a passive stress state. Therefore, the lateral pressure coefficient should be taken as follows:

$$
K_{p}=\frac{1+\sin \phi_{i}}{1-\sin \phi_{i}}
$$


That is, when the material is flowing, its deformation state and stress state should be different due to the height of material. The stress state of material from top to bottom gradually varies from the active state to the passive state. As the Bert test said, the lateral pressure coefficient $k(y)$ should be a function of material height. Assume that $k(y)$ is a conic as follows:

$$
k(y)=a y^{2}+b
$$

where $a, b$ are unknown constants, which can be determined by means of boundary conditions as follows:

$$
\begin{aligned}
& y=0, \quad k(y)=K_{a}, \\
& y=H, \quad k(y)=K_{p} .
\end{aligned}
$$

Thus, the lateral pressure coefficient of material in a certain depth could be decided by the following equation:

$$
k(y)=\frac{K_{p}-K_{a}}{H^{2}} y^{2}+K_{a}
$$

Substituting formula (8) into (3), expanding the integral term by Taylor series, and substituting into the boundary conditions $y=0, q=0$, the approximate equation for the dynamic pressure of bulkhead can be expressed as

$$
\begin{aligned}
& p=\rho g\left(\frac{K_{p}-K_{a}}{H^{2}} y^{2}+K_{a}\right) e^{-\left(m y^{3}+n y\right)} \\
& \cdot\left[y+\frac{n}{2} y^{2}+\frac{n^{2}}{6} y^{3}+\left(\frac{m}{4}+\frac{n^{2}}{24}\right) y^{4}+\frac{m n}{5} y^{5}\right. \\
&+\frac{m n^{2}}{12} y^{6}+\frac{m^{2}}{14} y^{7}+\frac{m^{2} n}{16} y^{8}+\frac{m^{2} n^{2}}{36} y^{9} \\
&\left.+\frac{m^{3}}{60} y^{10}+\frac{m^{3} n}{66} y^{11}+\frac{m^{2} n^{2}}{144} y^{12}\right]
\end{aligned}
$$

where $m=(2 f / R)\left(\left(K_{p}-K_{a}\right) / 3 H^{2}\right), n=(2 f / R) K_{a}$.

When the bulk material flows in the filling bin, the dynamic pressure against bulkhead at different depth could be calculated by (9). Minimum backpressure is the dynamic pressure on the bulkhead made by the material at the exit.

2.2. The Minimum Backpressure. In the construction, the most commonly used cement mortar is mixed by the volume ratio of $1: 3$, and its density is about $2100 \mathrm{~kg} / \mathrm{m}^{3}$; its mixture friction angle is about $29^{\circ}$, and the friction coefficient between bulk material and steel is 0.5 .

According to the geometry of backpressure filling system, the inlet hopper radius $R$ is $0.16 \mathrm{~m}$, the exit part corresponding backpressure depth is $y=0.715 \mathrm{~m}$, and total depth of packaging machine materials is $H=0.766 \mathrm{~m}$. Using (9), the dynamic pressure value, which is the minimum backpressure, could be achieved:

$$
P_{1}=3237.5(\mathrm{~Pa})
$$

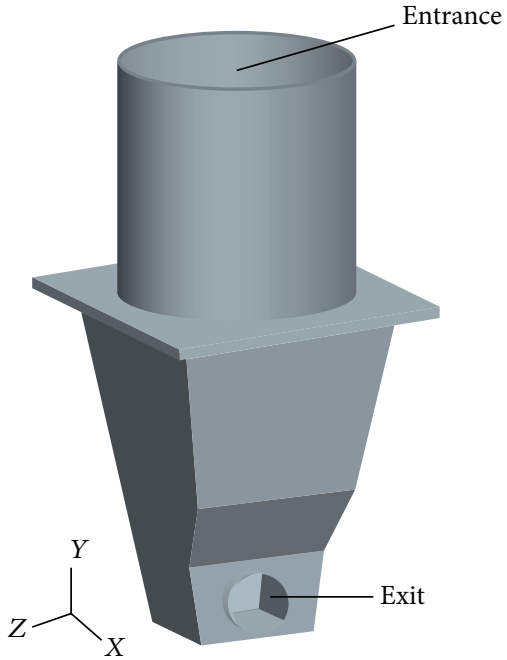

FIGURE 3: Backpressure filling system model.

2.3. The Maximum Backpressure. Considering the cement mortar is in a relatively dense state in the filling bin, it can be assumed incompressible. When the material suffers the backpressure that is greater than its own weight, the material will break down. Therefore, the maximum backpressure of the depth $y$ is the pressure value generated by the weight of the material at the same depth:

$$
p=\rho g y \text {. }
$$

Thus, when the depth of material exit $y$ is $0.715 \mathrm{~m}$, the density of material is $\rho=2100 \mathrm{~kg} / \mathrm{m}^{3}$, and the acceleration of gravity is $9.8 \mathrm{~m} / \mathrm{s}^{2}$, the maximum backpressure value is given as follows:

$$
P_{2}=14508.9(\mathrm{~Pa})
$$

\section{The Exit Velocity of Material with Free Flowing}

3.1. Theoretical Analysis of Exit Velocity of Material. Cement mortar material can be regarded as incompressible viscous fluid when it flows with free flowing under gravity in filling bin. When backpressure is not provided, material flowing can be considered as laminar flow due to its slow flow rate. The motion equations (Navier-Stokes equations) of incompressible viscous fluid under $Y$ direction in cylindrical coordinate system could be given as follows [5]; see Figure 3:

$$
\begin{aligned}
F_{y} & -\frac{1}{\rho} \frac{\partial p}{\partial y}+\mu\left(\frac{\partial^{2} u_{y}}{\partial r^{2}}+\frac{1}{r} \frac{\partial u_{y}}{\partial r}+\frac{1}{r^{2}} \frac{\partial^{2} u_{y}}{\partial y^{2}}\right) \\
& =\frac{\partial u_{y}}{\partial t}+u_{r} \frac{\partial u_{y}}{\partial r}+\frac{u_{\theta}}{r} \frac{\partial u_{y}}{\partial \theta}+u_{y} \frac{\partial u_{y}}{\partial y},
\end{aligned}
$$

where $u_{y}, u_{r}, u_{\theta}$ are velocity components of three coordinate directions. $\mu$ is the kinematic viscosity of bulk materials, $\rho$ is the density of material, $p$ is the pressure, and $F_{y}$ is the unit mass force of material under $Y$ direction. 
Because the material flowing is laminar and symmetry flow $u_{y}$ is independent of $Y$ and $\theta$ coordinate, $u_{y}=u(r$, $\theta, y)=u(y)$.

When the flowing is constant, the unit mass force $F_{y}=0$, and $\partial u_{y} / \partial t=0$. Equation (13) could be simplified as follows:

$$
\frac{\partial p}{\partial y}=\mu\left(\frac{\partial^{2} u}{\partial r^{2}}+\frac{1}{r} \frac{\partial u}{\partial r}\right)=\frac{\mu}{r} \frac{d}{d r}\left(r \frac{d u}{d r}\right) .
$$

$u_{y}$ is independent of $Y$ coordinate, so $\partial p / \partial y$ is constant along $Y$ direction. Assume that

$$
\frac{\partial p}{\partial y}=\frac{\Delta p}{L}=J \rho
$$

where $J$ is hydraulic slope and $\rho$ is the density of material.

By using boundary conditions $r=r_{0}, u=0$, the distribution of material velocity at exit could be achieved:

$$
u=\frac{\rho J}{4 \mu}\left(r_{0}^{2}-r^{2}\right)
$$

It indicates that for incompressible viscous flow the velocity distribution on any fracture surface is a revolving paraboloid whose mean flow velocity is a half of the maximum flow velocity $\left(v=u_{m} / 2\right)$.

When $r=0$, the velocity gets the maximum:

$$
u_{m}=\frac{\rho J}{4 \mu} r_{0}^{2}
$$

where $J=\lambda v^{2} / 4 r_{0} g, v$ is mean flow rate of exit, radius of exit $r_{0}=45 \mathrm{~mm}$, and $\lambda=\operatorname{tg} \phi_{i}$ is on-way resistance coefficient. The apparent viscosity of cement mortar is about $10-15 \mathrm{cp}$. If $12 \mathrm{cp}$ was picked, the dynamic viscosity could be as follows:

$$
\mu=12 \times 10^{-3} \times 9.8=0.1176 \mathrm{~kg} / \mathrm{m} \cdot \mathrm{s} .
$$

Substituting relevant parameters into (16), the velocities with no backpressure could be achieved as follows:

mean flow velocity of exit surface $v \approx 0.0794 \mathrm{~m} / \mathrm{s}$;

maximum velocity of exit surface $u_{m} \approx 0.159 \mathrm{~m} / \mathrm{s}$.

3.2. Numerical Simulation of Material Exit Velocity. Computational fluid dynamics (CFD) method by FLUENT software is used to simulate the exit velocity of bulk material in the backpressure filling system. FLUENT provides a flexible grid feature, and a variety of complex meshes can be classified easily via the structured grid and unstructured mesh area. For the three-dimensional problem, it can provide many grid units including tetrahedron, hexahedron, pyramid, wedge, and mixed grids. Besides, FLUENT also allows users to refine or roughen partial meshes according to their own solving scale, accuracy, and efficiency. For a flow area with the large gradient, FLUENT with adaptive characteristic of grid can give the solution with high accuracy [6-8].

Unstructured tetrahedral meshes with excellent adaptability could be selected when simulating the exit velocity of materials in the free flow process. According to the actual

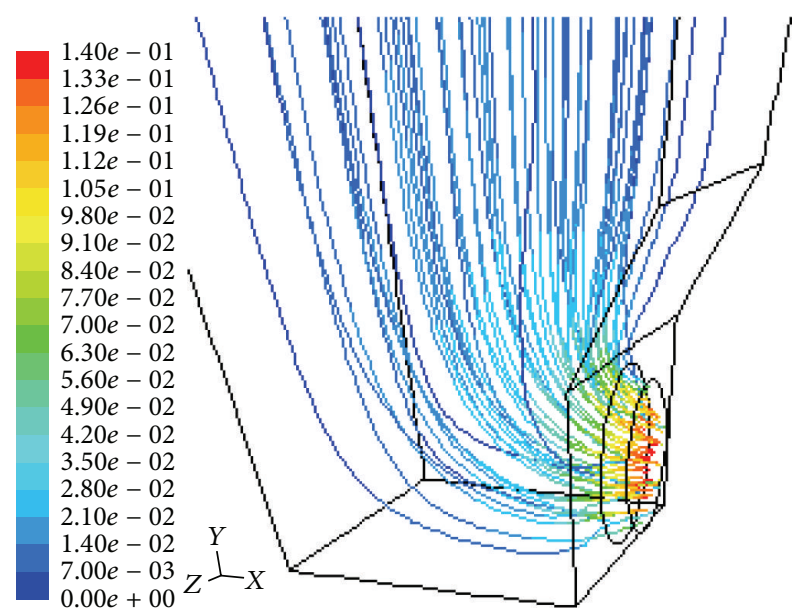

FIgURE 4: The velocity change near the exit.

flow ability of mortar, the 3D separator implicit solver will be selected. And the operating environment includes two parts; one is the selection of the reference pressure, and the other is the option of gravity. According to the operating environment of the cement mortar packaging machine, the atmosphere pressure, which is $101,325 \mathrm{~Pa}$, should be selected as the reference pressure, due to the direct contact with atmosphere at the inlet and outlet of the machine. Besides, standard pressure uses a discrete format. Momentum equation, turbulent kinetic energy equation, and turbulent kinetic energy dissipation rate equations use the secondorder discrete format, calculation of the pressure and velocity fields uses SIMPLEC algorithm, and the convergence criteria are set as $10^{-3}$. As the material flows by gravity, the gravity acceleration, which is $g=9.8 \mathrm{~m} / \mathrm{s}^{2}$, is set, and the direction is the negative $Y$-axis. The viscous laminar flow model (laminar model) is selected from among single-phase flow models, and the physical parameters of the material are also set. The materials inlet condition is selected as the boundary conditions of import velocity, and the material export condition is set as the boundary conditions of pressure outlet [9-12].

After dividing the mesh and setting computing model, material, and boundary conditions, the following settings should be clear in order to better control the solution process: select a discrete format, set underrelaxation factor, and initialize field variables and activate the monitoring variables. According to the flow characteristics of materials and the requirements of solution accuracy, the residual error of fluid computation shows that the convergence effect is good after more than two thousand iterations.

The velocity change near the exit could be as shown in Figure 4 when materials flow steadily in the filling bin.

As shown in Figure 4, cement mortars from different lays do not blend with each other in the flow, which accords with laminar flow standards. In the area with significant change of geometry size, flow line also changes significantly. At the same time, the material in the filling bin is not flowing rapidly, while the velocity at the exit is gradually increased. 


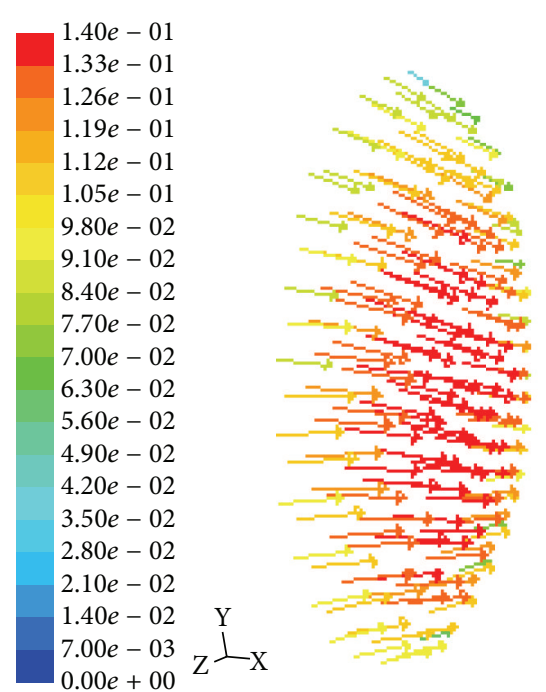

FIGURE 5: Velocity vector graph at exit.

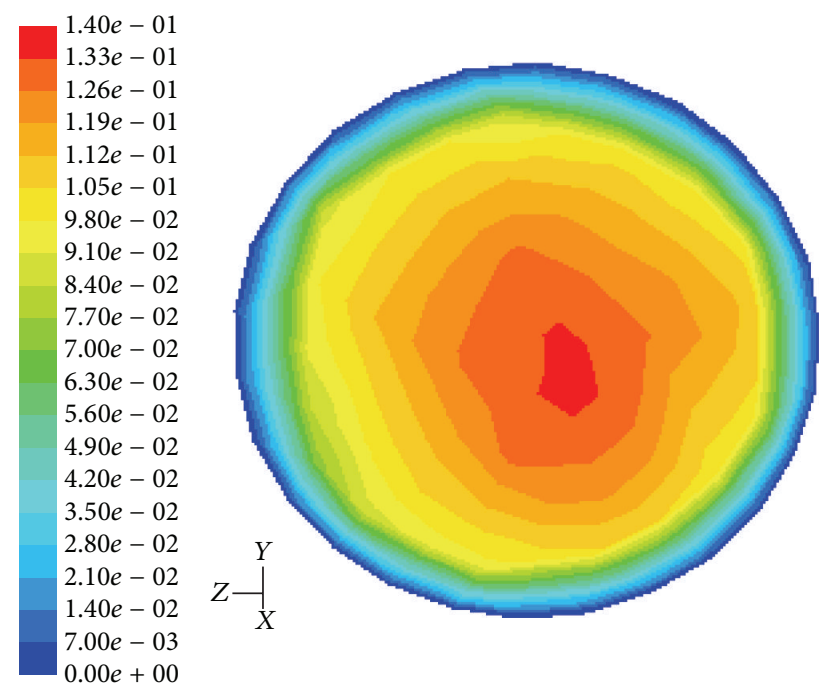

FIGURE 6: Velocity contour graph at exit surface.

Figure 5 is a velocity vector graph at the exit, the arrow color indicates the velocity, and the direction of the arrow indicates the direction of velocity. According to the analysis of CFD, the average velocity of exit is 0.0891 , whose error is about $10 \%$ when compared with the theoretical results, which is 0.0794 . Thus, the results of simulating are close to the theoretical calculation results, which means the models and parameters set in FLUENT are reasonable.

Figure 6 is the velocity contour graph at exit surface, where the maximum flow velocity is about $1.4 \mathrm{~m} / \mathrm{s}$, and the velocity along bulkhead is almost zero. It accords with flow regularity of bulk material.

According to the result of analog computation, the average velocity of exit $v \approx 0.0891 \mathrm{~m} / \mathrm{s}$, and the mass flow rate $q=1.267 \mathrm{~kg} / \mathrm{s}$. Therefore without the backpressure

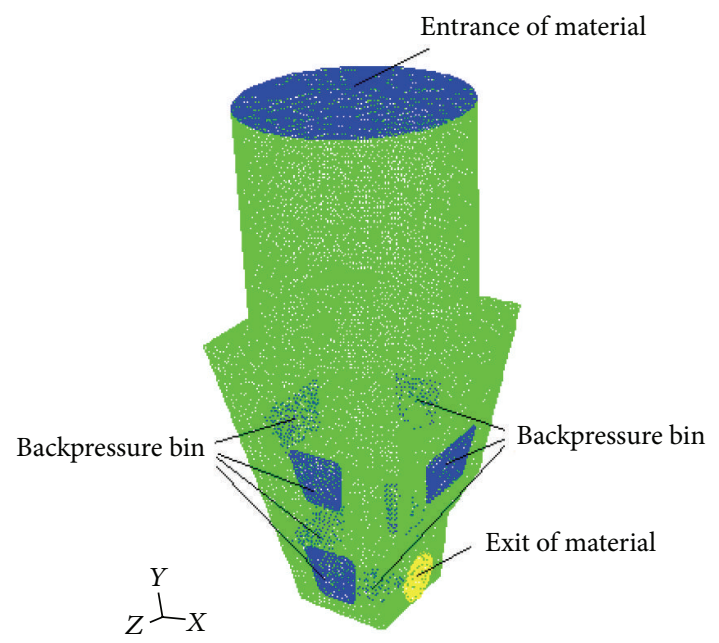

FIGURE 7: Geometric modeling and meshing with multiple backpressures coexisting.

the production capacity for a rotary packaging machine with 8 spouts could be achieved as follows:

$$
Q=8 q t=\frac{8 \times 1.267 \times 3600}{1000}=36.49(\mathrm{t} / \mathrm{h}) .
$$

\section{CFD Calculations of Exit Velocity under Backpressure Condition}

When the backpressure filling mechanism works, backpressure would have a huge impact on the velocity of material flow. Quantitative analysis on the effect of backpressure change to material flow velocity and finding the relationship between capacity of equipment and backpressure value will be beneficial to the equipment testing and working. This part of the study will be based on the previous research.

According to the real working conditions and the requirements of simple model principal, the following assumptions about materials and flow conditions are assumed.

(i) The flow of material is stable, which is steady-state flow.

(ii) The density of cement mortar mixture does not change with time.

(iii) The temperature and the energy of the flow would be ignored.

4.1. The Selection of the Physical Model of Multiphase Flow. For flow of mortar mixture inside the hopper, single-phase flow analysis, which worked on numerical simulation on gravity free flow, can be taken when the backpressure is zero; on the contrary, when considering the effect of backpressure, the Euler two-phase model on Euler-Euler numerical method can be selected because it is impossible to apply the pressure to the inlet part without inflow of materials in the FLUENT. In practice, the air inside the filling bin would never flow forward with the materials as the entrance of backpressure could only provide the pressure. Thus, the Euler model that 

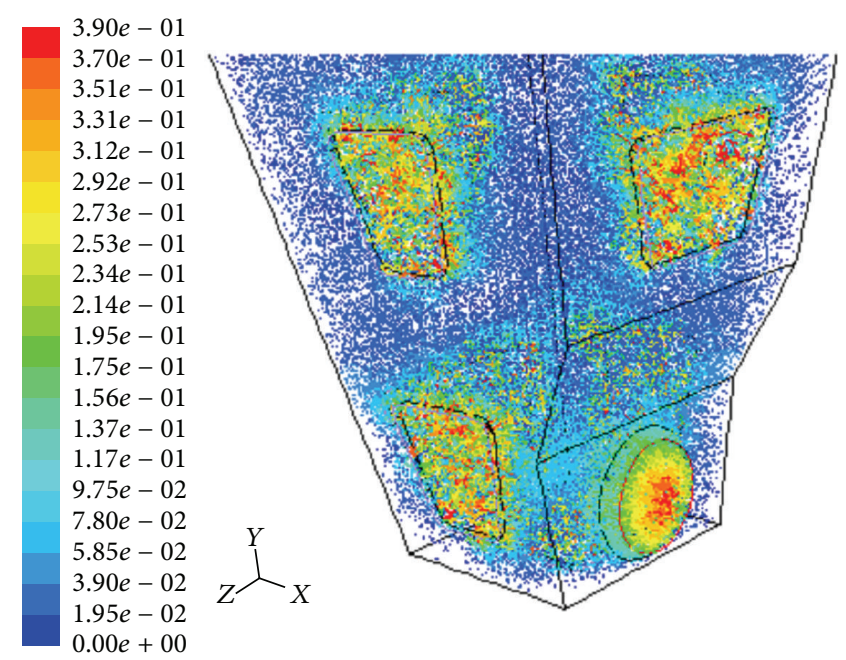

(a)

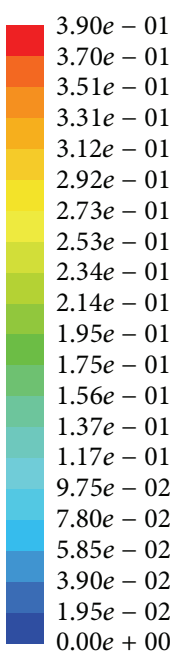

$.00 e+00$

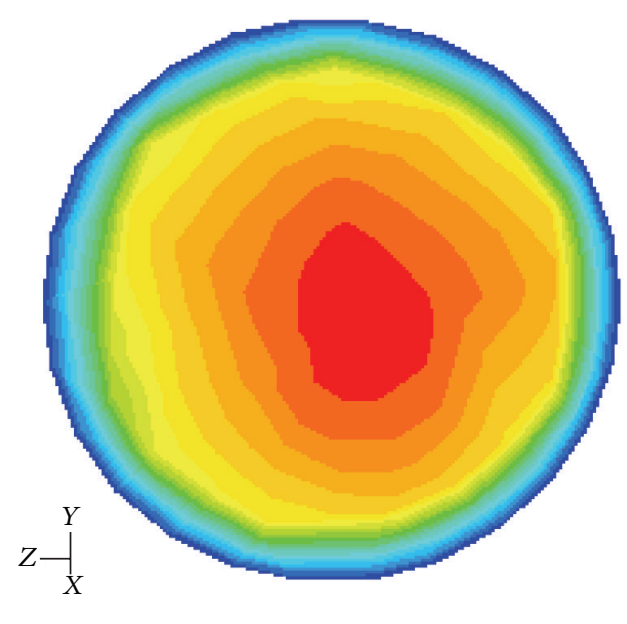

(b)

FIGURE 8: The distribution and contours of exit velocity when the backpressures are $12 \mathrm{kPa}$.

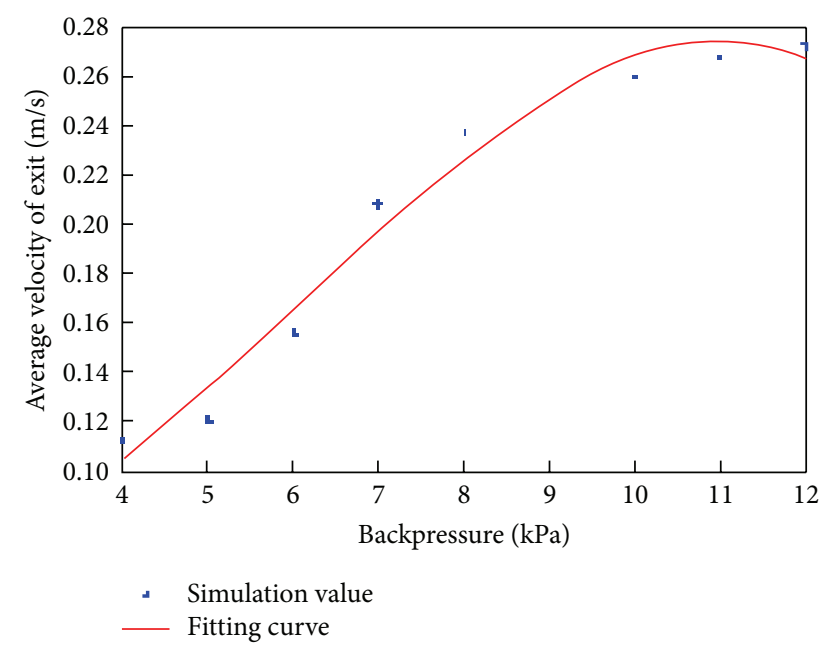

FIGURE 9: The fitting curve on average velocity of exit.

worked in this case could be simplified as the air loss ratio is zero [13-15].

4.2. Geometric Modeling and Meshing. When analyzing the impact of multiple backpressures on the bulk material flow, the mesh generation could be meshed according to the result above. Figure 7 expresses the geometry modeling and meshing with multiple backpressures coexisting. The adaptive and unstructured tetrahedral grids are used while meshing.

$3 \mathrm{D}$ implicit segregated solver is selected after the twophase model of Euler developing and boundary settings. During the simulating, standard discrete format is used to provide the pressure. The momentum equation, turbulent kinetic energy equation, and turbulent kinetic energy dissipation rate equations use second-order discrete format, the solving of

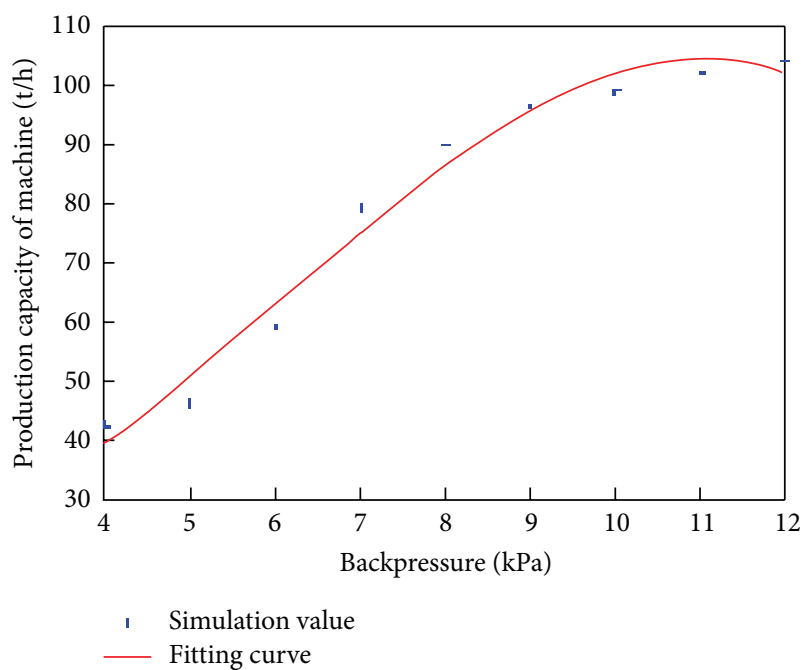

FIGURE 10: The fitting curve on production capacity of packaging machine.

pressure and velocity fields uses SIMPLEC algorithm, and the convergence criteria are set as $10^{-4}$.

4.3. Simulation Results and Analysis under Multiple Backpressures. To simulate the real working conditions of the filling mechanism, the situation when eight backpressures work together is simulated. Figure 8 shows the velocity distribution and velocity contours when the backpressures are $12 \mathrm{kPa}$.

As shown in Figure 8, due to the lifting and pushing of backpressures, the frictional resistance between bulkhead and bulk material reduced; it accelerated the material flow and then disordered the flow direction on a certain degree. However, because of the role of gravity, it is a mainstream for bulk material to flow towards the exit spout. The stable 
TABLE 1: The specific simulating results under different backpressures.

\begin{tabular}{lccc}
\hline $\begin{array}{l}\text { Backpressure } \\
(\mathrm{kPa})\end{array}$ & $\begin{array}{c}\text { Average } \\
\text { velocity of } \\
\text { exit }(\mathrm{m} / \mathrm{s})\end{array}$ & $\begin{array}{c}\text { Mass flow rate } \\
\text { of exit }(\mathrm{kg} / \mathrm{s})\end{array}$ & $\begin{array}{c}\text { Production } \\
\text { capacity of } \\
\text { machine }(\mathrm{t} / \mathrm{h})\end{array}$ \\
\hline 4 & 0.112 & 1.473 & 42.42 \\
5 & 0.120 & 1.603 & 46.17 \\
6 & 0.155 & 2.058 & 59.27 \\
7 & 0.208 & 2.759 & 79.46 \\
8 & 0.237 & 3.130 & 90.14 \\
9 & 0.252 & 3.342 & 96.25 \\
10 & 0.260 & 3.445 & 99.22 \\
11 & 0.268 & 3.555 & 102.38 \\
12 & 0.273 & 3.622 & 104.31 \\
\hline
\end{tabular}

backpressure in backpressure bin has significant influence on the flow ability of material.

In order to acquire the specific simulating results under different backpressures, the data processing software MATLAB is used to display the statistics shown in Table 1. The curve fitting is used to further simulation results, and fitting results are shown in Figures 9 and 10.

The relationship between average velocity of exit and backpressure is as shown in the following equation:

$$
\begin{aligned}
v= & -4.571 \times 10^{-4} P^{3}+8.39 \times 10^{-3} P^{2}-1.872 \\
& \times 10^{-2} P+7.346 \times 10^{-2} \quad\left(R^{2}=0.9791\right) .
\end{aligned}
$$

The relationship between production capacity of packaging machine and backpressure is as shown in the following equation:

$$
\begin{aligned}
Q= & -0.1662 P^{3}+3.005 P^{2}-5.656 P \\
& +24.55 \quad\left(R^{2}=0.9807\right) .
\end{aligned}
$$

In the practical work of rotary packaging machine, provided backpressure by backpressure filling mechanism should meet the requirements of packaging capacity. At the same time, it cannot break down material or let gases get into the cement bags or burst bags. Thus, the balance between the two requirements should be found to ensure production efficiency and the safety of production process.

As shown in Table 1 and Figure 10, when the backpressure is $7 \mathrm{kPa}$, the production capacity is low, which is about $80 \mathrm{t} / \mathrm{h}$. When the backpressure is $8 \mathrm{kPa}$, the production capacity is relatively high, which is around $90 \mathrm{t} / \mathrm{h}$ or more. When the backpressure is more than $10 \mathrm{kPa}$, production capacity of the equipment increases slowly as the pressure goes up; particularly when backpressure reaches $12 \mathrm{kPa}$ as Figure 8 showed, the flow of bulk material exhibits a certain degree of disorder. To ensure reliable operation of the equipment, the backpressure of the filling mechanism should be controlled within $11 \mathrm{kPa}$.

According to the theoretic calculation and CFD numerical analysis above, when rotary cement mortar packing
TABLE 2: The comparison on the simulation results and actual production capacity of machine.

\begin{tabular}{lcc}
\hline \multirow{2}{*}{ Backpressure $(\mathrm{kPa})$} & \multicolumn{2}{c}{ Production capacity of machine $(\mathrm{t} / \mathrm{h})$} \\
Simulating result & Actual result \\
\hline 6 & 59.27 & 54.50 \\
8 & 90.14 & 86.25 \\
10 & 99.22 & 97.45 \\
11 & 102.38 & 101.70 \\
12 & 104.31 & 105.60 \\
\hline
\end{tabular}

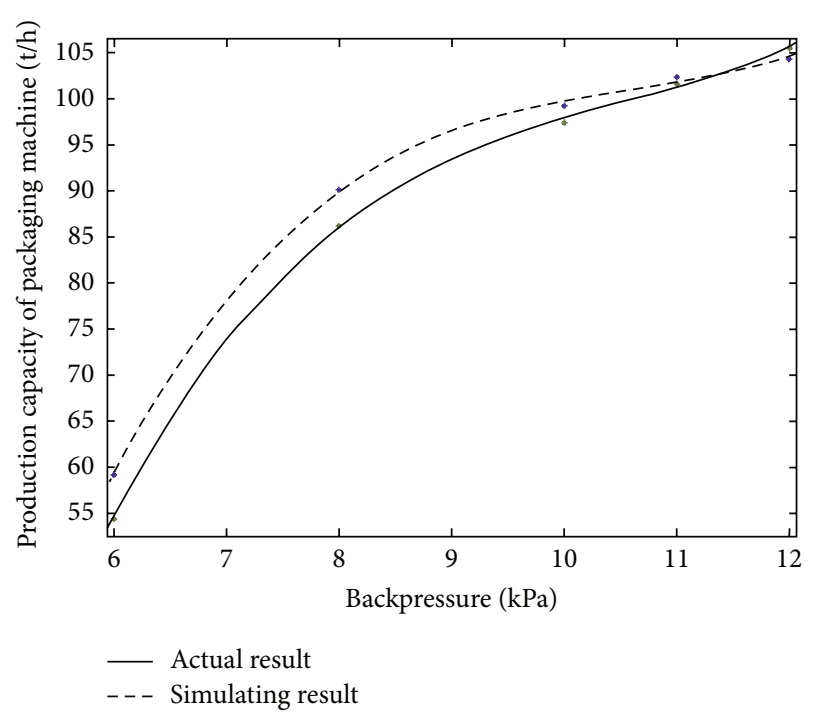

FIGURE 11: The fitting curve of production capacity based on simulating and actual result.

machine adopts backpressure filling system, the backpressure should be controlled at $8 \mathrm{kPa} \sim 11 \mathrm{kPa}$. In this way, the production capacity of equipment could be $90 \mathrm{t} / \mathrm{h} \sim 102 \mathrm{t} / \mathrm{h}$.

To verify the results of CFD simulation, the research set up an actual test on a rotary cement mortar packaging machine with backpressure filling system and recorded its actual production capacity under different backpressures. Table 2 shows these data and puts the CFD simulation results together.

Figure 11 shows that the actual result and the simulation result are very close. And rotary cement mortar packaging machine in the pilot running and following customer using has proved that the theoretical analysis results are accurate enough. That is, when the backpressure is controlled at about $11 \mathrm{kPa}$, production capacity of packaging equipment could be $100 \mathrm{t} / \mathrm{h}$.

\section{Conclusion}

The research is aimed at working principle of new backpressure filling system and acquired the range of backpressure of filling system by theoretical analysis. The physical models of multiphase flow based on CFD simulation for filling system are developed. The relationship between backpressure and 
exit velocity of the material is found, and finally production capacity of equipment to backpressure curve is achieved. Thus, the backpressure filling system can be manufactured and tested. It has been verified by practical production, and the relationship curve matched the operation of equipment well. The production capacity of packaging equipment could achieve $90 \sim 100 \mathrm{t} / \mathrm{h}$, when the backpressure is controlled at $8 \mathrm{kPa} 11 \mathrm{kPa}$.

The new filling system can be applied not only to dry mortar powder, but also to cement, flour, and other pure powders. The CFD models could be applied on the computer simulation for filling system. When the backpressure is beyond the maximum backpressure, the CFD model is no longer applicable. In the future work, according to the material properties, new suggestions on backpressure control could be given, and, based on this, the optimization of filling bin could be achieved.

\section{Conflict of Interests}

The authors declare that there is no conflict of interests regarding the publication of this paper.

\section{Acknowledgments}

This research was supported in part by a Grant-in-Aid for 44th Scientific returned people start funding from the Education Ministry of China and Six Talent Peaks Funding Program of Jiangsu Province.

\section{References}

[1] J. Qian, H. P. Cai, J. P. Lu et al., "The backpressure filling device on mortar packaging machine," Chinese patent no. 201220259089.X, 2012.

[2] H. Sakaguchi and E. Ozaki, "Basic research for the plugging of granular flow," Journal of the Society of Powder Technology, vol. 30, no. 3, pp. 183-187, 1993.

[3] Y.-J. Ma and J. Qian, "Numerical simulation of the cementsand mix flowing in a storage hopper," Packaging and Food Machinery, vol. 26, no. 4, pp. 28-32, 2008.

[4] Y.-J. Ma, The Study on Working Principle of Back Pressure Filling System for Mortar Packer, Jiangnan University, Wuxi, China, 2008.

[5] Z. Han and W. Yu, "Evaluation of flow ability of powder materials in cement plants," ZKG International, no. 12, pp. 694699, 1996

[6] R. Manikantan and E. James Gunasekaran, "Modeling and analysing of air filter in air intake system in automobile engine," Advances in Mechanical Engineering, vol. 2013, Article ID 654396, 6 pages, 2013

[7] S.-Y. Cho, K.-Y. Ahn, Y.-D. Lee, and Y.-C. Kim, "Optimal design of a centrifugal compressor impeller using evolutionary algorithms," Mathematical Problems in Engineering, vol. 2012, Article ID 752931, 22 pages, 2012.

[8] H. Massah and L. Oshinowo, "Advanced gas-solid multiphase flow models offer significant process inprovments," Fluent Software Users, vol. 15, no. 3, pp. 1-6, 2000.

[9] Fluent, User Defined Function Manual, Fluent, New York, NY, USA, 2003.
[10] Z. Zuo, S. Liu, Y. Fan, and Y. Wu, "Optimization of a centrifugal boiler circulating pump's casing based on CFD and FEM analyses," Advances in Mechanical Engineering, vol. 2014, Article ID 532892, 10 pages, 2014.

[11] Fluent Inc, GAMBIT Modeling Guide, Fluent Inc, 2003.

[12] M. Kalinkevych and O. Shcherbakov, "Numerical modeling of the flow in a vaneless diffuser of centrifugal compressor stage," ISRN Mechanical Engineering, vol. 2013, Article ID 602384, 9 pages, 2013.

[13] Fluent, User's Guide, Fluent, Lebanon, NH, USA, 2003.

[14] X. Song, M. Cao, W. Shin, W. Cao, S. Kang, and Y. Park, "Numerical investigation of a liquid-gas ejector used for shipping ballast water treatment," Mathematical Problems in Engineering, vol. 2014, Article ID 259593, 7 pages, 2014.

[15] S. Shojaee, S. H. Hosseini, and B. S. Razavi, "Computational fluid dynamics simulation of multiphase flow in structured packings," Journal of Applied Mathematics, vol. 2012, Article ID 917650, 17 pages, 2012. 


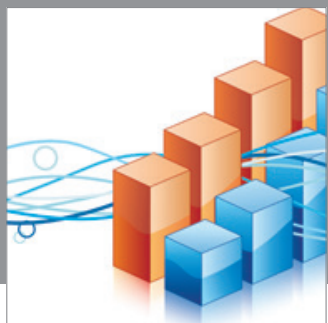

Advances in

Operations Research

mansans

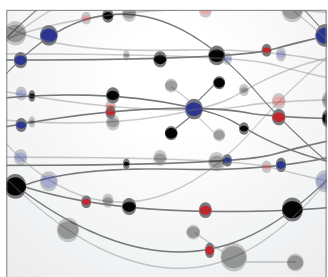

The Scientific World Journal
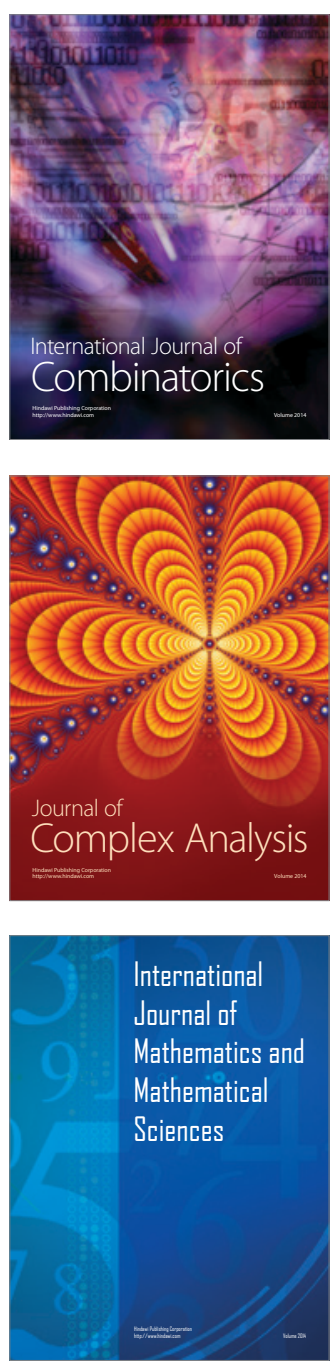
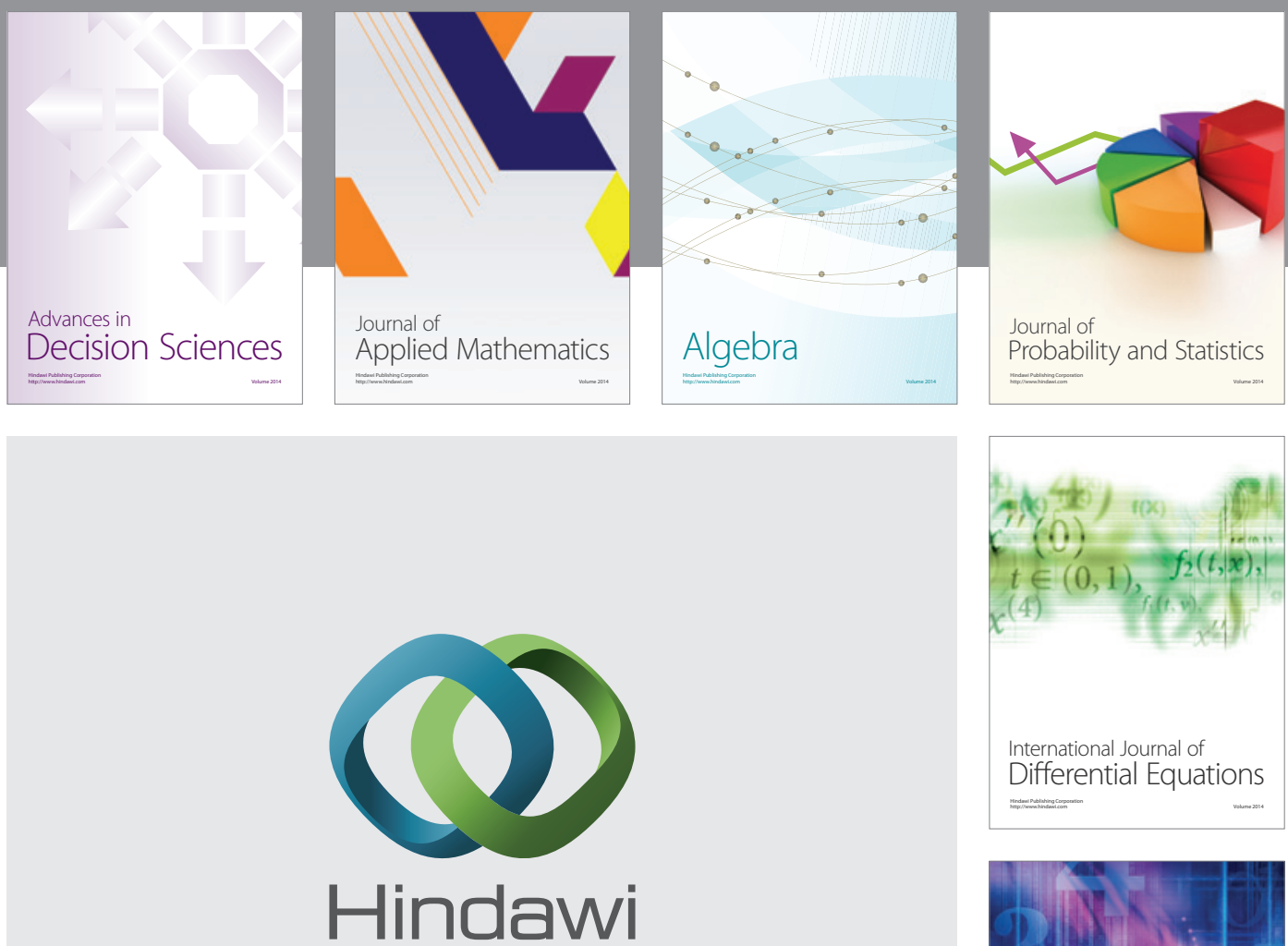

Submit your manuscripts at http://www.hindawi.com
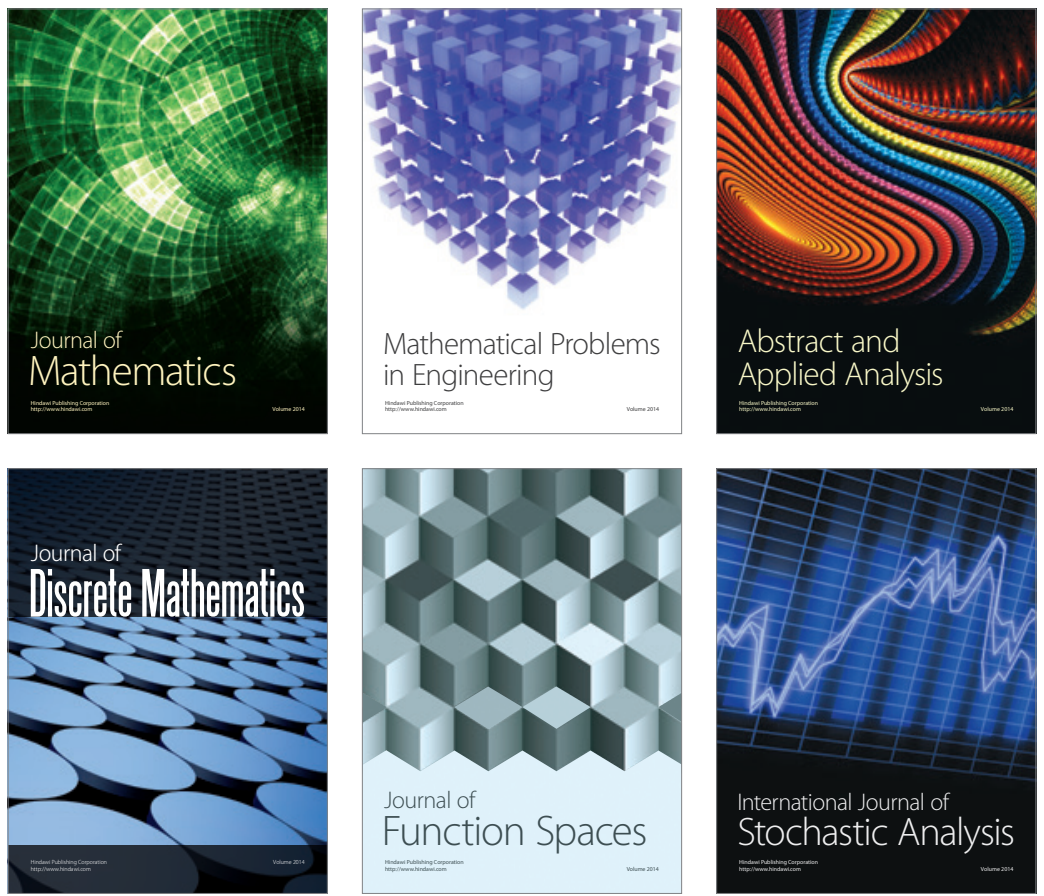

Journal of

Function Spaces

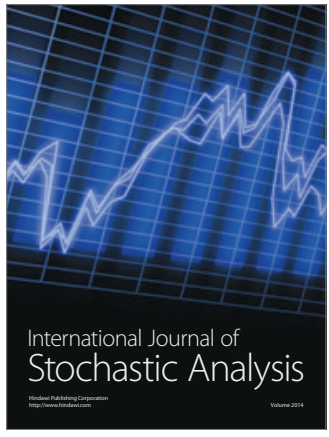

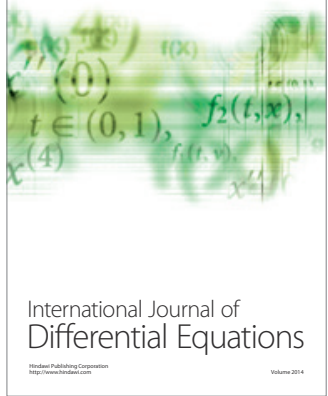
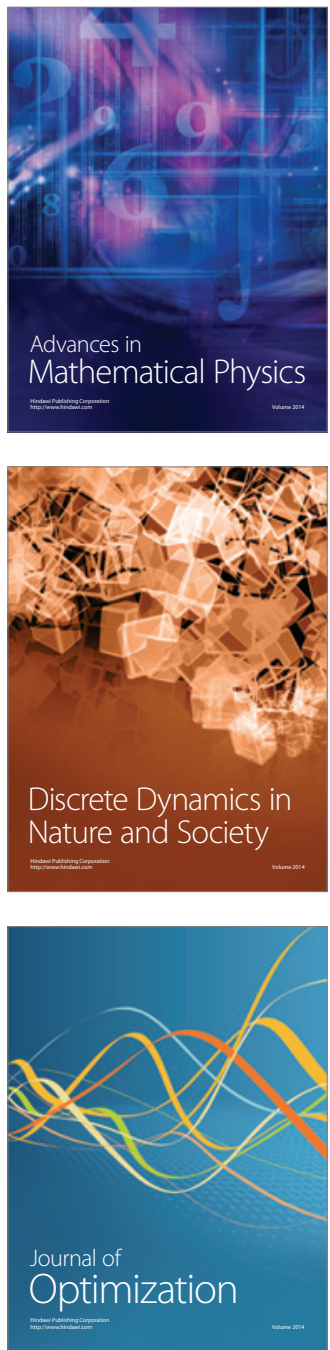